\title{
Breast tuberculosis: analysis of 24 patients
}

\author{
๑DTibel Tuna ${ }^{1}$, @Serdar Coşkun ${ }^{2}$ \\ ${ }^{1}$ Ondokuz Mayıs University, Faculty of Medicine, Department of Chest Diseases, Samsun, Turkey \\ ${ }^{2}$ Samsun Gazi State Hospital, Samsun, Turkey
}

Cite this article as: Tuna T, Coşkun S. Breast tuberculosis: analysis of 24 patients. J Health Sci Med 2022; 5(1): 88-93.

\begin{abstract}
Introduction: Breast tuberculosis is a disease that is difficult to diagnose with its clinical appearance and can be confused with breast abscesses and breast carcinomas. In this study, we aimed to analyze 24 patients diagnosed with breast tuberculosis, followed up and treated.

Material and Method: Among 4489 patients examined in the breast diseases outpatient clinic between January 2012 and December 2020, patients diagnosed with breast tuberculosis were evaluated retrospectively. Demographic, clinical features, radiological findings, histopathological and microbiological findings of the patients were recorded. Diagnosis, treatment protocols and treatment outcomes were evaluated.

Results: A total of 24 breast tuberculosis cases were analyzed in the study. The mean age was 41.6 (28-64 years) and all patients were diagnosed for the first time. There was a history of oral contraceptive use in 10 patients. Among patients, 19 of them were in the reproductive age, and five patients were in the postmenopausal period. Four patients were in lactating period. On physical examination, breast mass was detected in 16 (67\%) patients, abscess was present in 5 patients (20.8\%), and sinus and discharge were evident in three patients (12.5\%). Breast ultrasonography revealed a breast mass in 16 patients $(67 \%)$, abscess in eight patients (33\%), and axillary lymphadenopathy in six patients (25\%). The diagnosis was made by core biopsy in 10 (41.6\%) patients, fine-needle aspiration biopsy in three patients (12.5\%), and incisional biopsy in 11 patients (45.8\%). All patients were given quadruple antituberculosis therapy in the first 2 months for 9 months, and double antituberculosis therapy for 7 months. In addition to antituberculosis treatment, mass excision was performed in three patients (12.5\%), segmental mastectomy in one patient (4.1\%), simple mastectomy in one patient (4.1\%), and abscess drainage were completed in eight patients (33.3\%). Cure was achieved in all patients after treatment.

Conclusion: Breast tuberculosis is a rare disease that can be difficult to diagnose unless the disease itself is suspected. As the diagnosis of the disease is delayed, the disease becomes complicated and the need for surgical treatment increases as well as medical treatment. Although there are different opinions about the duration of the treatment, in our study, cure was achieved with 9 months of treatment and no recurrence was observed in the 1-year follow-up. Since 10 patients had a history of oral contraceptive use, further research is needed to understand whether oral contraceptive use is a predisposing factor in the development of breast tuberculosis.
\end{abstract}

Keywords: Extrapulmonary tuberculosis, diagnosis, management

\section{INTRODUCTION}

Tuberculosis is a global problem in the world and the second-leading infectious killer after COVID-19 in 2020. It is a disease that is associated with the death of 1.5 million people annually (1). Tuberculosis can infect many organs other than the lungs. Breast tuberculosis is a very rare form of extrapulmonary tuberculosis (2). Breast tuberculosis was first described by Sir Astley Cooper in 1829 (3). Breast tuberculosis is seen more commonly in developing countries, and since the main treatment of breast tuberculosis is antituberculous therapy, it is important to differentiate breast tuberculosis from other granulomatous diseases of the breast and breast cancer. The incidence of breast tuberculosis has been reported as $0.2 \%-6.8 \%$ (mean $1.7 \%$ ), while in Western societies, it is reported as below $0.1 \%$ (2). This is explained by the fact that the breast tissue is resistant to M. tuberculosis (4).

Breast tuberculosis is generally classified as primary or secondary (5). It does not have a clinically apparent appearance. It can be confused with other breast diseases, breast abscesses and breast carcinomas. Since the most common manifestation is painful or painless 
masses, it is difficult to diagnose breast tuberculosis with its clinical appearance. Other manifestations may include diffuse breast swelling, edema, nipple retraction, fistulization, multiple sinuses, skin ulcers, and recurrent abscesses (6-8).

Different diagnostic methods are available for breast tuberculosis, however, clinical suspicion is the first step in the diagnosis. Radiological tests (ultrasound examination, computed tomography, magnetic resonance and mammography) are not specific to achieve a diagnosis. Biological tests (culture, Ziehl-Neelsen staining) are of low sensitivity and time-consuming (2). Polymerase chain reaction (PCR) has high sensitivity, however, it has a higher cost and is not affordable for regions where tuberculosis is endemic (9). Fine needle aspiration biopsy is useful in making the differential diagnosis, but it is difficult to distinguish between granulomatous mastitis and sarcoidosis (10). Histopathological examination with biopsy of breast sections reveals granulomatous inflammation and caseification necrosis (7). The treatment of breast tuberculosis is carried out by applying medical therapy and accompanying surgery. Anti-tuberculosis treatment is usually given for 6 months or longer (11). In this study, we aimed to examine the demographic information, diagnosis and treatment methods of 24 patients who were diagnosed, followed up and treated in a chest diseases hospital in 9 years.

\section{MATERIAL AND METHOD}

The study was carried out with the permission of Ondokuz Mayis University Clinical Research Ethics Committee (Date: 25.03.2021, Decision No: 2021/146). All procedures were carried out in accordance with the ethical rules and the principles of the Declaration of Helsinki.

Between January 2012 and December 2020, 24 patients with breast tuberculosis were evaluated retrospectively, out of 4489 patients examined in the breast diseases outpatient clinic. Demographic, clinical features, radiological findings, histopathological and microbiological findings of the patients were recorded. In the demographic evaluation of the patients, age, gender, educational status, and place of residence were recorded. Clinical features were evaluated as admission symptoms, location and appearance of the lesion, presence of axillary lymphadenopathy, lactation status, pre or postmenopausal status, use of oral contraceptives, and presence of concomitant disease. Breast ultrasound, mammography and chest X-ray were performed radiologically. Histopathologically, core needle biopsy, fine-needle aspiration biopsy, and incisional biopsy were performed and granulomatous inflammation including caseification necrosis was observed in patients.
Microbiologically acid staining, Ziehl Nielsen staining and culture cultivation were performed and results were recorded. These patients who were diagnosed with breast tuberculosis received antituberculosis treatment for a total of 9 months, the first 2 months being quadruple with isoniazid (INH), rifampin (RIF), ethambutol (ETM), pyrazinamide (PZA), and the remaining 7 months with INH and RIF. The results of control appointment of the patients at the $12^{\text {th }}$ month after diagnosis were evaluated. Data were computerized using SPSS v26.0 (Chicago, IL) and descriptive analyzes were presented as percentage and mean (minimum-maximum).

\section{RESULTS}

A total of 24 breast tuberculosis cases were examined. All of the patients were female. The mean age was 41.6 (2864 years) and all patients were diagnosed with primary breast tuberculosis. Of the patients, nine (37.5\%) lived in rural areas and $15(62.5 \%)$ lived in the city center. Fourteen patients $(58.3 \%)$ were from the working class and 10 of them $(41.7 \%)$ were from the civil servant class. Seven patients $(29.1 \%)$ had graduate education, and seventeen patients (70.8\%) had undergraduate education. There was a history of oral contraceptive use in 10 patients $(41.7 \%)$. While thirteen patients $(54.1 \%)$ did not report any comorbidity, 11 patients $(45.8 \%)$ had additional disease ( 5 patients had diabetes mellitus, two patients had hypertension, one patient had diabetes mellitus and hypertension, two patients had rheumatoid arthritis, one patient had ankylosing spondylitis). The sociodemographic characteristics of the patients were shown in Table 1.

\begin{tabular}{|lcc|}
\hline \multicolumn{2}{|c|}{ Table 1. Sociodemographic characteristics } \\
\hline \multicolumn{2}{|c|}{$(\%)$} & N \\
\hline Age & $\begin{array}{c}\text { Mean 41.6 } \\
\text { (min 28-maks 64) }\end{array}$ & 24 \\
Gender & & \\
$\quad$ Female & $100.0 \%$ & 24 \\
$\quad$ Male & $0.0 \%$ & 0 \\
Marital status & & \\
$\quad$ Married & $83.3 \%$ & 20 \\
$\quad$ Single & $16.7 \%$ & 4 \\
Profession & & \\
$\quad$ Farmer & $33.3 \%$ & 8 \\
$\quad$ Worker & $12.5 \%$ & 3 \\
$\quad$ Housewife & $12.5 \%$ & 3 \\
Officer & $41.6 \%$ & 10 \\
Educational level & & \\
$\quad$ Primary school & $25.0 \%$ & 6 \\
Middle school & $20.8 \%$ & 5 \\
High school & $25.0 \%$ & 6 \\
$\quad$ University & $29.1 \%$ & 7 \\
Region of origin & & 9 \\
Rural area & $37.5 \%$ & 15 \\
\hline City center & $62.5 \%$ & \\
\hline
\end{tabular}


Among patients, 19 (79.2\%) were in the reproductive age, and five $(20 \%)$ patients were in the postmenopausal period. Four (16.6) patients were currently lactating. Breast mass was detected in $16(66.7 \%)$ patients, abscess in five $(20.8 \%)$, and sinus and discharge in three (12.5\%) patients. Lesions were located in the left breast in 11 (45.8\%) patients, and in the right breast in $13(54.2 \%)$ patients. The localization of the lesions in the breast was in the middle outer quadrant in nine cases $(37.5 \%)$, in the upper outer quadrant in 12 cases (50\%), and in the periareolar area in four cases $(16.5 \%)$. Clinical features were shown in Table 2.

Breast ultrasonography was performed in all patients. A breast mass was detected in $16(67 \%)$ patients, and abscess was detected in eight patients (33\%). Axillary lymphadenopathy was negative in 18 patients $(75 \%)$ and axillary lymphadenopathy was present in six patients (25\%) on examination. Mammography was performed in nine patients (37.5\%), and no specific finding was found. Biopsy, culture, and clinical response were evaluated to achieve a diagnosis. The core needle biopsy was performed in 10 patients (41.6\%), fine needle aspiration biopsy in three patients (12.5\%), and incisional biopsy in 11 patients $(45.8 \%)$. Histological specimens showed epithelioid cell granulomas with caseous necrosis. While no growth was observed in the culture in 17 (70.8\%) of the patients, the culture result was positive in seven patients $(29.1 \%)$. The diagnostic methods and findings are summarized in Table 3.

After diagnosis, 11 patients (45.8\%) were given antituberculosis treatment without any further procedure. In addition to antituberculosis treatment, mass excision was performed in three patients (12.5\%), segmental mastectomy in one patient (4.1\%), simple mastectomy in one patient (4.1\%), and abscess drainage in eight patients (33.3\%) (Table 4). All patients were given quadruple therapy (INH, RIF, PZA, ETB) in the first two months for nine months, and double therapy (INH, RIF) for seven months. Cure was achieved in all patients after treatment. Treatment applications were shown in Table 4.

\section{DISCUSSION}

In this study, we evaluated the diagnosis, follow-up and treatment results of patients with breast tuberculosis, which is a rare form of tuberculosis.

Tuberculosis is a common disease in endemic areas, however, solitary breast tuberculosis is rarely seen since the breast is more resistant to tuberculosis than other organs. Differentiation of granulomatous lesions (fungal, sarcoidosis, syphilis, plasma cell mastitis, actinomyces) encountered in the breast is necessary, and histopathological examination is required to

\section{Table 2. Clinical characteristics}

\begin{tabular}{|c|c|c|}
\hline & $(\%)$ & $\mathbf{N}$ \\
\hline \multicolumn{3}{|l|}{ Menopause } \\
\hline Premenopausal & $79.1 \%$ & 19 \\
\hline Postmenopausal & $20.9 \%$ & 5 \\
\hline \multicolumn{3}{|l|}{ Location } \\
\hline Right breast & $54.1 \%$ & 13 \\
\hline Left breast & $45.8 \%$ & 11 \\
\hline \multicolumn{3}{|l|}{ Location in breast } \\
\hline Upper outer & $50.0 \%$ & 12 \\
\hline Middle outer & $37.5 \%$ & 9 \\
\hline Periareolar & $16.6 \%$ & 4 \\
\hline \multicolumn{3}{|l|}{ Risk factors } \\
\hline Multiparity & $50.0 \%$ & 12 \\
\hline History of tuberculosis & $4.1 \%$ & 1 \\
\hline Lactation & $16.6 \%$ & 4 \\
\hline \multicolumn{3}{|l|}{ Presence of comorbidities } \\
\hline Yes & $45.9 \%$ & 11 \\
\hline No & $54.1 \%$ & 13 \\
\hline \multicolumn{3}{|l|}{ Lactation } \\
\hline Yes & $16.6 \%$ & 4 \\
\hline No & $83.3 \%$ & 20 \\
\hline \multicolumn{3}{|l|}{ Lung x-ray } \\
\hline Normal & $84.4 \%$ & 21 \\
\hline Sequale & $16.6 \%$ & 3 \\
\hline
\end{tabular}

\section{Table 3. Diagnostic methods and results}

\begin{tabular}{|c|c|c|}
\hline & $\%$ & $\mathbf{N}$ \\
\hline \multicolumn{3}{|l|}{ Diagnostic methods } \\
\hline Fine needle aspiration biopsy & $12.5 \%$ & 3 \\
\hline Core needle biopsy & $41.6 \%$ & 10 \\
\hline Incisional biopsy & $45.8 \%$ & 11 \\
\hline Positive culture & $29.1 \%$ & 7 \\
\hline \multicolumn{3}{|l|}{ Clinical examination findings } \\
\hline Mass & $66.6 \%$ & 16 \\
\hline Abscess & $20.8 \%$ & 5 \\
\hline Sinus & $12.5 \%$ & 3 \\
\hline \multicolumn{3}{|l|}{ Ultrasound findings } \\
\hline Abscess & $20.8 \%$ & 5 \\
\hline Mass & $66.6 \%$ & 16 \\
\hline Axiller lymphadenopathy & $25.0 \%$ & 6 \\
\hline
\end{tabular}

\begin{tabular}{|lcc|}
\hline Table 4. Treatment methods & \% & N \\
\hline Treatment & & \\
$\quad$ Antituberculosis therapy & $100.0 \%$ & 24 \\
$\quad$ Antituberculosis therapy + surgery & $54.1 \%$ & 13 \\
Surgical treatment & & \\
$\quad$ None & $45.8 \%$ & 11 \\
Drainage & $33.3 \%$ & 8 \\
Segmental mastectomy & $4.1 \%$ & 1 \\
Simple mastectomy & $4.1 \%$ & 1 \\
Excision & $12.5 \%$ & 3 \\
\hline
\end{tabular}


distinguish it from malignant lesions. In this singlecenter retrospective study which analyzed the rare cases of breast tuberculosis demographically, clinically and therapeutically; it was observed that a diagnosis of breast tuberculosis can be achieved after initially suspecting from tuberculosis. It was also seen that the treatments given for at least 9 months were more effective and there was no recurrence observed in the annual follow-up.

Although breast tuberculosis can be seen in any age group, breast tuberculosis is rare in the elderly and individuals under the age of $18(7,12,13)$ in the literature, while it is more common in women of reproductive age $(14,15)$. In our study, $20.8 \%$ of the patients were in the postmenopausal period. The incidence of tuberculous mastitis increases in lactation, and $16.6 \%$ of our patients were in the lactation period. Enlargement and increase in vascularity in the breast canals during pregnancy and lactation cause infection (16). In particular, pregnancy predisposes to infection by suppressing the T-helper 1 pro-inflammatory response (17). Immunosuppressive use and HIV infection are among risk factors for primary and reactivation tuberculosis (18). None of the patients had HIV positivity. However, one patient was using TNF-alpha blocker with the diagnosis of Ankylosing spondylitis, and 1 patient was using steroids for rheumatoid arthritis. It was noteworthy that 10 of our patients $(41.7 \%)$ had a history of oral contraceptive use. In a case-control study, Haleh et al. reported that multiparity, lactation duration, and the rate of use of oral contraceptives were significantly higher in patients with idiopathic granulomatosis mastitis compared to controls (19). In their retrospective study, Prasad et al. indicated that $54.79 \%$ of patients with idiopathic granulomatosis mastitis reported a history of oral contraceptive use without evidence of tuberculosis (20). This has raised doubts whether oral contraceptive use is a predisposing factor in the development of breast tuberculosis, however, further research is needed to demonstrate such an association.

In the process leading to the diagnosis of breast tuberculosis, the duration of the symptoms and the clinical course vary. While the time from symptom onset to diagnosis is a few weeks in western societies, this period is more than 7 months in eastern societies and Africa $(8,21,22)$. It was observed that our patients presented within 4-6 weeks after the onset of symptoms, and were diagnosed within 8-10 weeks. It was thought that the socioeconomic status of the patients and the fact that they came from rural areas are factors in this delay. The most common symptom was a breast mass. Apart from this, abscesses and discharge were also seen. In our cases, $66 \%$ mass, $20.8 \%$ abscess and $12.6 \%$ sinus (discharge) were present. The diagnosis of breast tuberculosis was probably delayed due to the prescription of empirical antibiotic therapy as the first step in treatment for patients with abscess and discharge.

Although breast tuberculosis can be seen in both breasts simultaneously, it is very rare. The incidence rates in the right or left breast are not superior to each other (2325). In our study, we did not detect simultaneous cases in both breasts, and there was no significant difference between the right and left breasts in terms of the presence of tuberculosis. In the literature review by Gianluca et al. (26), periareolar involvement was observed as $17.3 \%$, similarly in our study, lesions were detected in the upper outer quadrant of the breast at a rate of $50 \%$, in the middle outer quadrant of $37.5 \%$, and in the periareolar region at a rate of $16.6 \%$. In the same review, less than $10 \%$ of the patients were reported to have concomitant active pulmonary tuberculosis. In our study, there was no accompanying pulmonary tuberculosis, but 3 patients $(16.6 \%)$ had previous tuberculosis sequelae.

The clinical appearance of the disease can be confused with many diseases. It is most often confused with fibroadenoma of the breast because the most common finding of fibroadenoma is a mass $(27,28)$. It can also be confused with granulomatous and inflammatory diseases such as idiopathic granulomatous mastitis, sarcoidosis, wegener's granulomatosis, giant cell arteritis and other infectious diseases such as brucella, actinomyces, fungal infections and fat necrosis (27-29). Since there is no gold standard method for diagnosis, the diagnosis is made by demonstrating the microorganism and/or histopathologically. In some cases, the diagnosis is made by showing the response to antituberculosis treatment and pathology (31-33). While $71.9 \%$ of our patients were diagnosed with the pathological examination, microorganisms were only shown in $29.1 \%$ of the cases, similar to the low rate of diagnosis by showing microorganisms in the literature (6). The success rate of diagnosis by PCR has been reported as 50\% (26), however, PCR diagnosis rates could not be evaluated in this study since this application was not performed in our cases

The most important diagnostic method for breast tuberculosis is histopathological examination. In the literature, the diagnosis rate was reported as $64 \%$ with fine-needle aspiration biopsy and 93\% with biopsy (26), however, in our study, the rate of diagnosis was $12.5 \%$ with fine-needle aspiration biopsy, $41.6 \%$ with core needle aspiration biopsy and $45.8 \%$ with incisional biopsy. The reasons for the low diagnosis rate with fineneedle aspiration biopsy in this study were the presence of insufficient material and the lack of sufficient experience for cytological examination. The failed attempts with fine needle aspiration biopsy were completed with core needle or incisional biopsy. 
There is no standard guideline for treatment. The mainstay of treatment consists of antituberculous therapy. While some physicians recommend 6 months of standard antituberculosis treatment, some authors recommend 9 months of antituberculosis treatment (2 months quadruple 7 months dual treatment). Although there are opinions that suggest 9-months of treatment has no extra contribution (34), there are publications reporting that the relapse rate was less with 9 months of treatment $(33,35)$.

In our patients, recurrence was not observed in the 1-year follow-up of patients with 9 months of treatment. In breast tuberculosis, patients who usually require surgery are complicated cases that develop abscess and sinus formation and present to clinics late after the symptoms. Patients who underwent radical surgery have been reported as $4.6 \%$ in the literature (subtotal or total mastectomy) (8). The rate of radical surgery in our patients was $8.2 \%$ (segmental and simple mastectomy). Our patients who underwent radical surgery were diagnosed late and their breast tissue was destroyed. While minor surgical applications (abscess drainage, aspiration, sinus resection, necrotic tissue resection) were applied as $11.3 \%$ in the literature, it was applied to $33 \%$ of our patients. The reason for the higher rate of minor surgical procedures in our study compared to the literature is that they were performed for diagnostic purposes (26).

\section{Limitations}

Our study has some limitations. Firstly, the study was planned retrospectively. Since all patients were treated for 9 months, 9 vs 6-month treatment regimens were not compared. The patients' results at 12 th month after 9 months of treatment were evaluated in this study. Longer follow-up could have provided clearer information in terms of follow-up results of recurrence. Since there was no cytologist in our hospital, fine needle aspiration biopsy could not be fully utilized for diagnostic purposes.

\section{CONCLUSION}

In conclusion, breast tuberculosis is still a rare disease that can be difficult to diagnose unless the disease itself is suspected, despite the increasing diagnostic and therapeutic possibilities. The most important point in diagnosis is to suspect the disease first. As the diagnosis of the disease is delayed, the disease becomes complicated and the need for surgical treatment increases as well as medical treatment. Thus, as the diagnosis is delayed, it becomes more difficult to protect the breast, and psychological and social effects increase after breast surgery in women of reproductive age. It was noteworthy that 10 patients $(41.7 \%)$ had a history of oral contraceptive use, however, whether oral contraceptive use is a predisposing factor in the development of breast tuberculosis needs further investigations. Although there are different opinions on the duration of the treatment, no recurrence was observed in 9 months of treatment at 1-year follow-up. Therefore, we suggest that 9 months of treatment is ideal to prevent recurrence.

\section{ETHICAL DECLARATIONS}

Ethics Committee Approval: The study was carried out with the permission of Ondokuz Mayıs University Clinical Research Ethics Committee (Date: 25.03.2021, Decision No: 2021/146).

Informed Consent: Because the study was designed retrospectively, no written informed consent form was obtained from patients.

Referee Evaluation Process: Externally peer-reviewed.

Conflict of Interest Statement: The authors have no conflicts of interest to declare.

Financial Disclosure: The authors declared that this study has received no financial support.

Author Contributions: All of the authors declare that they have all participated in the design, execution, and analysis of the paper and that they have approved the final version

\section{REFERENCES}

1. World Health Organization (WHO). Global tuberculosis report 2020. New York: WHO. 14 Oct 2020.

2. Baharoon S. Tuberculosis of the breast. Ann Thorac Med 2008; 3: $110-4$.

3. Cooper. Illustrations of the diseases of the breast, part I. London: Longman, Rees, Orme, Brown and Green, 1829: 73.

4. Kalac N, Ozkan B, Bayiz H. Brest tuberculosis. Breast 2002; 11 : 346-9.

5. Bouti K, Soualhi M, Marc K, et al. Postmenopausal breast tuberculosis-report of four cases. Breast Care (Basel) 2012; 7: 411-3.

6. Kessler E, Woolloch Y. Granulomatous mastitis: a lesion clinically simulating carcinoma. Am J Clin Pathol 1972; 58: 642-6.

7. Marinopoulos S, Lourantou D, Gatzionis T, Dimitrakakis C, Papaspyrou I, Antsaklis A. Breast tuberculosis: diagnosis, management and treatment. Int J Surg Case Rep 2012; 3: 548-50.

8. Khanna R, Prasanna GV, Gupta P, Kumar M, Khanna S, Khanna AK. Mammary tuberculosis: report on 52 cases.Postgrad Med J 2002; 78: 422-4.

9. Bani-Hani KE, Yaghan RJ, Matalka II, Mazahreh TS. Tuberculous mastitis: a disease not to be forgotten. Int J Tuberc Lung Dis 2005; 9: $920-5$.

10.Gupta D, Rajwanshi A, Gupta SK, Nijhawan R, Saran RK, Singh R. Fine needle aspiration cytology in the diagnosis of tuberculous mastitis. Acta Cytol 1999; 43: 191-4.

11.Tewari M, Shukla HS. Breast tuberculosis: diagnosis, clinical features and management. Indian J Med Res 2005; 122: 103-10.

12.Zandrino F, Monetti F, Gandolfo N. Primary tuberculosis of the breast. A case report. Acta Radiol 2000; 41: 61-3. 
13.Luh SP, Hsu JD, Lai YS, Chen SW. Primary tuberculous infection of breast: experiences of surgical resection for aged patients and review of literature. J Zhejiang Univ Sci B 2007; 8: 580-3.

14. Indumathi CK, Alladi A, Dinakar C, Rout PL. Tuberculosis of the breast in an adolescent girl: a rare presentation. J Trop Pediatr 2007; 53: 133-4.

15. Bianco SR, Gurgel RL, Tavares Mde A. Radiological aspects of primary tuberculosis in the breast: a case report and review of the literature. Rev Soc Bras Med Trop 2009; 42: 203-5.

16. Walker M. Conquering common breast-feeding problems. J Perinat Neonatal Nurs 2008; 22: 267-74.

17. Mathad JS, Gupta A. Tuberculosis in pregnant and postpartum women: epidemiology, management, and research gaps. Clin Infect Dis 2012; 55: 1532-49.

18. Korenromp EL, Scano F, Williams BG, Dye C, Nunn P. Effects of human immunodeficiency virus infection on recurrence of tuberculosis after rifampin-based treatment: an analytical review. Clin Infect Dis 2003; 37: 101-12.

19. Pluguez-Turull CW, Nanyes JE, Quintero CJ, et al. Idiopathic granulomatous mastitis: manifestations at multimodality imaging and pitfalls. Radiographics 2018; 38: 330-56.

20. Prasad S, Jaiprakash P, Dave A, Pai D. Idiopathic granulomatous mastitis: an institutional experience. Turk J Surg 2017; 33: 100-3.

21.Elsiddig KE, Khalil EA, Elhag IA, et al. Granulomatous mammary disease: ten years' experience with fine needle aspiration cytology. Int J Tuberc Lung Dis 2003; 7: 365-9.

22. Harris SH, Khan MA, Khan R, Haque F, Syed A, Ansari MM. Mammary tuberculosis: analysis of thirty-eight patients. ANZ J Surg 2006; 76: 234-7.

23. Van Keirsbilck J, Riphagen I, Struyven H, et al. Bilateral mammary tuberculosis associated with a borderline ovarian tumor. Breast Care 2008; 3: 200-3.

24. Ruiz-Moreno JL, Peña-Santos G. Bilateral tuberculous mastitis nulliparous patient, initially treated as idiopathic granulomatous mastitis. Ginecol Obstet Mex 2012; 80: 228-31.

25. Yanamandra U, Pathak N, Naithani N, Grover N, Nair V. Tuberculosis of breast: unusual manifestation of tuberculosis. J Infect Chemother 2012; 18: 109-11.

26. Quaglio G, Pizzol D, Isaakidis P, et al. Breast tuberculosis in women: a systematic review. Am J Trop Med Hyg 2019; 101: 12 21

27. Olu-Eddo AN, Ugiagbe EE. Benign breast lesion in an African population. Niger Med J 2011; 52: 211-6.

28. Amin AL, Purdy AC, Mattingly JD, Kong AL, Termuhlen PM. Benign breast disease. Surg Clin North Am 2013; 93: 299-308.

29. Nemenqani D, Yaqoob N. Fine needle aspiration cytology of inflammatory breast lesions. J Pak Med Assoc 2009; 59: 167-70.

30. Kataria SP, Sharma J, Singh G, Kumar S, Malik S, Kumar V. Primary breast mucormycosis: FNAC diagnosis of a rare entity. Diagn Cytopathol 2016; 44: 761-3.

31.Gupta D, Rajwanshi A, Gupta SK, Nijhawan R, Saran RK, Singh R. Fine needle aspiration cytology in the diagnosis of tuberculous mastitis. Acta Cytol 1999; 43: 191-4.

32. Kakkar S, Kapila K, Singh MK, Verma K. Tuberculosis of the breast. A cytomorphologic study. Acta Cytol 2000; 44: 292-6.

33. Jalali U, Rasul S, Khan A, Baig N, Khan A, Akhter R. Tuberculous mastitis. J Coll Physicians Surg Pak 2005; 15: 234-7.

34. Sinha R, Rahul. Breast tuberculosis. Indian J Tuberc 2019; 66: 6-11.

35. Shinde SR, Chandawarkar RY, Deshmukh SP. Tuberculosis of the breast masquerading as carcinoma: a study of 100 patients. World J Surg 1995; 19: 379-81. 